专家推介：本文通过研究不同碱腐蚀工艺对 MCP 通道内壁表面粗䊁度、碱金属离子浓度以及 MCP 增益 的影响, 并通过优化碱腐蚀工艺, 进一步提高了 MCP 的增益, 同时也降低了 MCP 的噪声系数。

\title{
碱腐蚀工艺对微通道板性能的影响研究
}

\author{
张正君，李婧雯，牛鹏杰，丛晓庆，邱祥彪，王 健 \\ （北方夜视技术股份有限公司南京分公司，江苏 南京 210006）
}

\begin{abstract}
摘要：本文探究了微通道板腐蚀工艺中碱溶液的腐蚀时间对其增益的影响。利用 X 射线光电子能谱 分析仪和原子力显微镜, 测试了不同碱腐蚀时间作用后微通道板通道内壁碱金属元素的含量变化情 况, 以及通道内壁粗精度变化趋势。结果表明, 在不影响微通道板通道内壁粗糙度的情况下, 一定 程度上减少碱腐蚀时间可以降低微通道板通道内壁的碱金属元素的流失，从而提高通道内部二次电 子发射能力, 提高微通道板增益。微通道板电性能以及制管后产品增益和噪声性能结果表明, 减少 碱腐蚀时间, 并未增加制管后噪声系数, 而通过提高微通道板内表面碱金属的含量, 有助于制管增 益的提高。
\end{abstract}

关键词: 微通道板; 腐蚀; 碱溶液; 碱金属; 增益

中图分类号：TN223 文献标识码：A 文章编号：1001-8891(2020)-08-0752-06

\section{Micro-channel Plate Etching Technology in Lye}

ZHANG Zhengjun, LI Jingwen, NIU Pengjie, CONG Xiaoqing, QIU Xiangbiao, WANG Jian

(Nanjing Branch, North Night Vision Technology Co., LTD., Nanjing 211106, China)

\begin{abstract}
In this study, the effect of lye corrosion time on the gain of a microchannel plate is investigated. By adjusting the lye-etching time, the concentration of alkali metals and the roughness of the channel inner wall are visualized via X-ray photoelectron spectroscopy and atomic force microscopy. Results show that reducing the lye-etching time can reduce the loss of alkali metal elements in the inner wall of the microchannel plate without affecting its roughness, thereby improving the secondary electron emission capacity and the microchannel plate gain. Further tests show that higher alkali metals can improve the gain of both the microchannel plates and image intensifier tubes, whereas the noise coefficient in the image intensifier tube does not increase with reduced lye-etching time.
\end{abstract}

Key words: micro-channel plate, etching, lye, alkali metals, gain

\section{0 引言}

微通道板 (micro-channel plate, MCP) 作为微光 像增强器的核心部件, 通过将二维空间分布的电子进 行倍增来实现信号放大并成像。MCP 广泛应用于离 子探测和高速摄像等领域 ${ }^{[1-4]}$ 。MCP 的性能参数主要 包括其增益、噪声系数以及空间分辨能力。通道发射 层二次电子发射系数直接影响 MCP 增益。而腐蚀和 氢还原是形成微通道板通道及电阻层、二次电子发射 层的过程 ${ }^{[5-6]}$ 。

采用酸蚀方法对 MCP 芯料玻璃进行去除, 形成
完整的通道是 MCP 生产过程的关键工序 ${ }^{[7-8]}$ 。芯料玻 璃的酸蚀速率要比皮料玻璃高出几个数量级。在 MCP 的压屏工艺阶段, 由于温度和压力的作用导致 芯皮之间相互渗透, 形成渗透层 ${ }^{[9]}$ 。渗透层的存在会 使得通道内壁在酸蚀后期形成致密的硅氧保护层, 从 而影响芯料的去除。碱溶液的参与有助于破坏硅氧保 护层, 进一步将芯料暴露出来, 待下一次进行酸蚀。 通过酸碱交替腐蚀的方式可以确保 MCP 通道的生 成，并降低内壁的粗粘度。

相对皮料玻璃中的其他成分来说，碱金属元素对 微通道板的增益贡献最为显著。使用酸碱溶液交替腐 
蚀渗透层的过程中有可能会造成皮料中原子半径小 的碱金属氧化物流失 ${ }^{[10]}$ 。为了提高 MCP 增益, 对腐 蚀工艺进行优化改进是相对有效的措施。在目前 MCP 腐蚀工艺的研究中, 碱蚀时间对于 MCP 的理化 性能研究少有涉及, 本文通过调整碱蚀时间来研究腐 蚀工艺中碱液对 MCP 性能的影响, 并将其对 MCP 制管后增益的影响进行评估。

\section{1 试验设计}

本文通过控制 MCP 腐蚀过程中碱蚀时长, 对通 道内壁的碱金属元素含量较高的工艺进行研究, 结合 MCP 内壁粗粘度和测试情况, 并对其性能进行初步 评估, 随后对制管后的增益与噪声进行测定, 作为工 艺可靠性的最终验证。

具体的腐蚀工艺对比如表 1 所示。酸选用浓度 为 $1 \mathrm{~mol} / \mathrm{L}$ 的稀盐酸; 碱为 $\mathrm{NaOH}$ 溶液, 质量浓度为 $5 \%$; 酸碱溶液的温度为 $25^{\circ} \mathrm{C}$ 。4 种腐蚀工艺都进行 两次酸碱交替。腐蚀采用磁力搅拌装置带动溶液旋 转。在酸碱交替之间采用纯水超声清洗工艺, 超声 波频率为 $40 \mathrm{kHz}$, 功率为 $300 \mathrm{~W}$ 。

\section{2 表征分析}

首先利用同一批段号的 MCP 进行了 4 种腐蚀工 艺试验, 每种工艺 20 片 MCP, 利用 XPS 对腐蚀后
通道内部元素的分布及含量进行了测定。图 1 反应 了对 4 种腐蚀工艺处理后的通道内壁的形貌结构。 可以看到经 4 种工艺处理后, 除了在样品制作过程 中带来的玻璃碎屑外, 4 组样片均形成了完整的通道 结构。

随后研究了腐蚀后 MCP 通道内壁的元素成分, 以工艺 2 处理的样品为例, 如图 2 所示。酸碱交替腐 蚀后的微通道板内部主要的元素包括 $\mathrm{O} 、 \mathrm{Si} 、 \mathrm{~Pb} 、 \mathrm{~K}$ 和 $\mathrm{Na}$ 等。经测定, 各组 MCP 内壁表面 $\mathrm{Si}$ 元素的含 量在 $30 \% \sim 35 \%, O$ 元素为 $50 \% \sim 55 \%, \mathrm{~Pb}$ 元素为 $20 \% \sim 25 \%, K$ 元素为 $4 \% \sim 5 \%, \mathrm{Na}<2 \%$ 。以 $\mathrm{K}$ 元 素为主的碱金属离子对 MCP 性能的影响相对明显。

4 种腐蚀工艺处理后的 MCP 内壁表面 K 元素和 $\mathrm{Na}$ 元素的能谱分布如图 3 所示, 可以看到 4 种腐蚀 工艺处理后的通道内壁碱金属含量分布相对一致, K 元素特征峰主要出现在 $293 \mathrm{eV}$ 和 $296 \mathrm{eV}$ 附近, Na 元素的特征峰主要出现在 $1071 \mathrm{eV}$ 附近。

在对 $\mathrm{K}$ 元素含量的测定结果中, 工艺 $1 \sim$ 工艺 4 分别为 $4.51 \% 、 4.82 \% 、 4.72 \%$ 和 $4.34 \%$ 。测定所得 $\mathrm{Na}$ 元素的含量, 工艺 $1 \sim$ 工艺 4 分别为 $1.7 \%$ 、 $1.9 \% 、 1.56 \%$ 和 1.5\%。考虑到碱金属元素对 MCP 性 能影响最大, 而工艺 2 与工艺 3 处理后的 MCP 内壁 $\mathrm{K} 、 \mathrm{Na}$ 元素整体含量较高, 因此本文对工艺 2、3 开 展了进一步研究。

表 1 不同腐蚀工艺设计

Table 1 The design of different etching processes

\begin{tabular}{ccccc}
\hline Etching Process & Step 1: Acid $1^{\text {st }}$ & Step 2: Lye $1^{\text {st }}$ & Step 3: Acid 2 & Step 4: Lye $^{\text {nd }}$ \\
\hline Process 1 & & & $10 \mathrm{~min}$ \\
Process 2 & \multirow{3}{*}{$60 \mathrm{~min}$} & $30 \mathrm{~min}$ & $60 \mathrm{~min}$ & $20 \mathrm{~min}$ \\
Process 3 & & & $30 \mathrm{~min}$ \\
Process 4 & & & $40 \mathrm{~min}$ \\
\hline
\end{tabular}

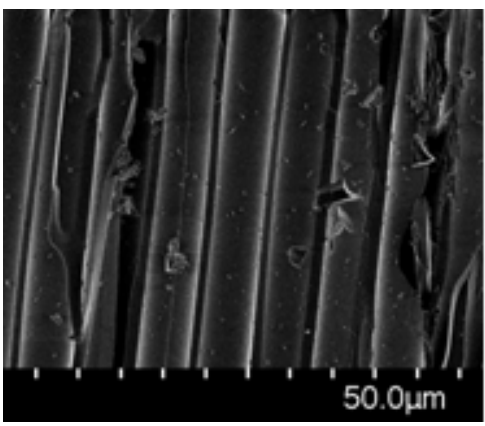

(a) Process 1

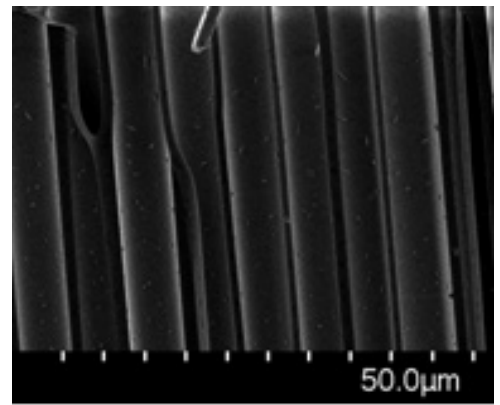

(b) Process 2 


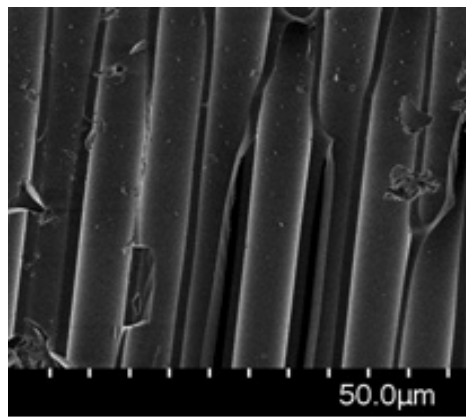

(c) Process 3

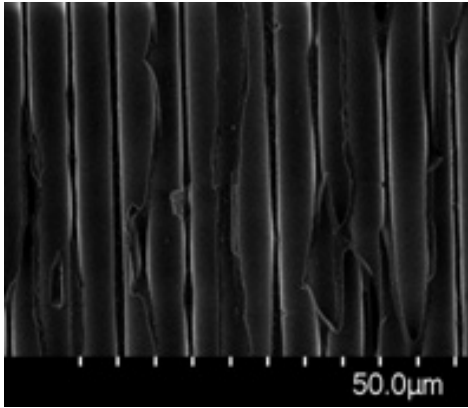

(d) Process 4

图 1 四种工艺在通道内壁的形貌情况

Fig.1 The appearances on the cliffs of MCP with different etching processes

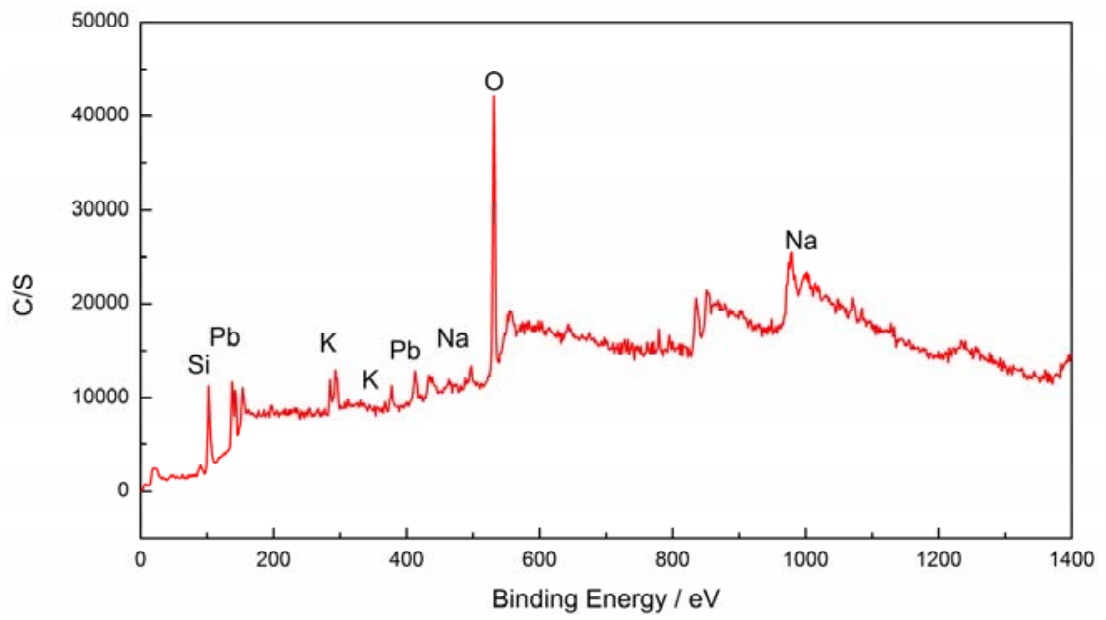

图 2 工艺 $2 \mathrm{MCP}$ 内壁元素分布情况

Fig.2 The distribution of elements of MCP with etching process 2

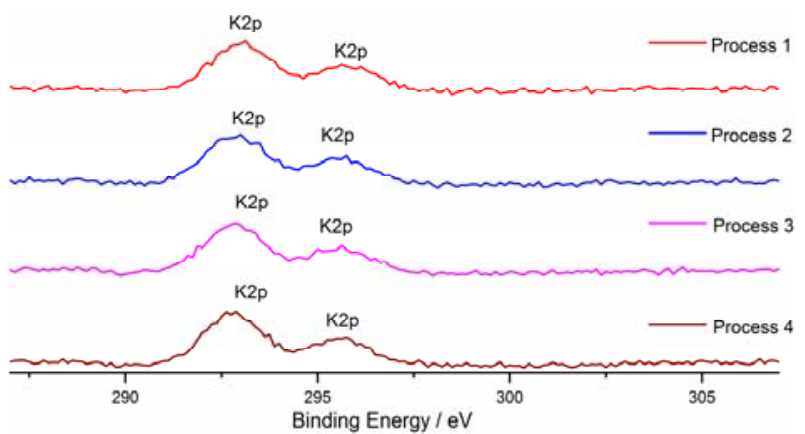

(a) Distribution of $\mathrm{K}$

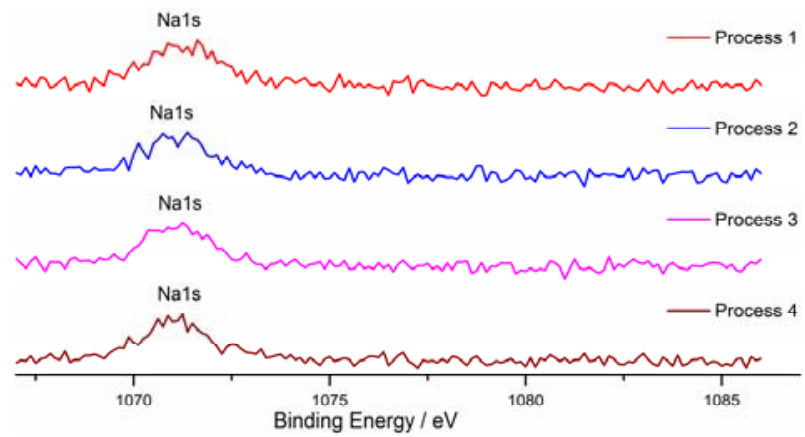

(b) Distribution of $\mathrm{Na}$

图 34 种工艺对应的 $\mathrm{K}$ 和 $\mathrm{Na}$ 元素窄谱分布图

Fig.3 The distributions of $\mathrm{K}$ and Na elements in four kinds of etching proceses

\section{3 结果与分析}

\section{1 不同碱蚀时长对通道内壁碱金属含量的影响}

本文通过 $\mathrm{Ar}$ 离子刻蚀与 XPS 能谱分析结合, 比 较了两种腐蚀工艺处理后的通道表层碱金属元素含 量在内壁的分布情况, 两种腐蚀工艺选用同一段号 各 30 片微通道板。图 4 反应了不同碱蚀时长下通道 内壁碱金属的平均含量随着 $\mathrm{Ar}$ 离子刻蚀时间的变化 情况。利用通道内壁材料的刻蚀与 XPS 交互作用, 可以获得其不同深度方向上的元素分布。可以看到 $\mathrm{K}$ 元素在通道内壁表面的含量要高于 $\mathrm{Na}$ 元素。随着 刻蚀深度的增加, 两种碱金属的含量大体呈现下降
的趋势。经过较短碱蚀工艺 (工艺 2, 第二次碱蚀时 长 $20 \mathrm{~min}$ ）处理的 MCP 内壁，两种碱金属含量要明 显高于较长碱蚀工艺（工艺 3, 第二次碱蚀时长 30 $\min ） 。$ 刻蚀 $1 \mathrm{~min}$ 后, 工艺 2 腐蚀通道内壁 $\mathrm{Na}$ 和 $\mathrm{K}$ 元素含量迅速下降。随着刻蚀时间的增加, 腐蚀工 艺 2 处理的 MCP 通道内壁 $\mathrm{Na}$ 元素在趋于平稳后出 现小幅上升, 而 $\mathrm{K}$ 元素则基本稳定在 4\%左右。正常 腐蚀通道内壁在不同刻蚀时间里, 两种碱金属元素 含量出现了小幅波动。就整体而言, 腐蚀工艺 2 的 两种碱金属元素的总含量均要高于腐蚀工艺 3 。

碱金属离子对二次电子发射系数的提高以及抑 制增益的衰减有显著的作用, 碱金属离子的存在可 
以降低通道内壁表面势垒, 电子更容易逸出。上述 结果表明适当减少碱腐蚀的时间有助于通道内壁中 的碱金属元素含量维持在较高的水平, 进而有助于 提高二次电子发射系数 ${ }^{[11-12]}$ 。

\section{2 碱蚀时长对通道内壁粗粘度的影响}

MCP 通道内壁经过酸碱腐蚀处理后会形成活性 表面, 对于后续除气工艺以及二次电子的发射性能 都有较大的影响, 通道内壁粗粘度越大, 活性表面 积也越大。酸碱交替腐蚀可以有效降低通道内壁粗 䊁度, 而碱蚀时间长短对粗糔度会造成一定的影 响。同时考虑到减少碱腐蚀时间后，芯皮渗透层可 能难以清除, 该渗透层的存在不仅会增加表面粗粘 度, 引起电子记忆效应 ${ }^{[10]}$, 而且会增加通道内壁气体 的吸附量, 进而带来离子噪声。本文利用原子力显微 镜对上述两种腐蚀工艺处理的 MCP 的内壁粗鋉度进 行了测定。

如图 5 所示, 从两种腐蚀工艺下通道内壁的形貌 并没有明显的差异, 两种腐蚀工艺的通道内壁粗粘度 测量平均值如表 2 所示。经两种碱蚀时间处理后，通 道内壁表面的均方根粗粘度分别达到了 $0.86 \mathrm{~nm}$ (工艺

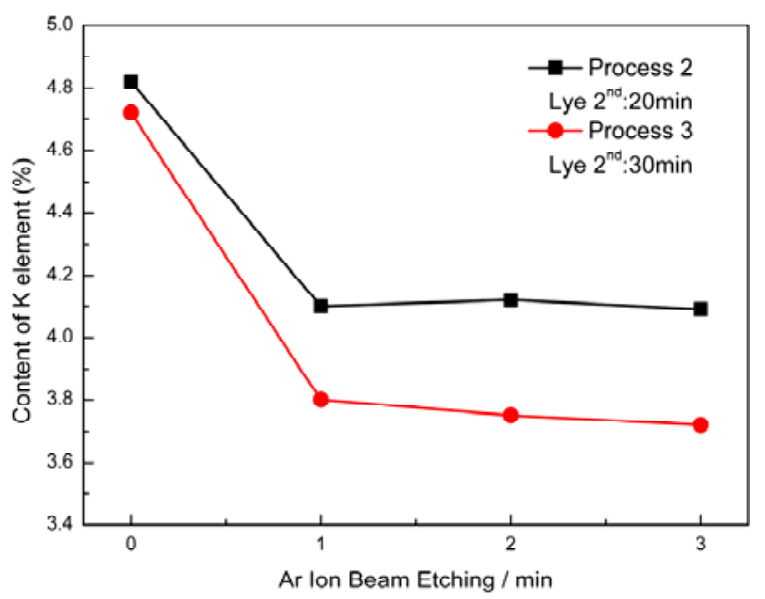

(a) Content distributions of K element
2）和 $0.8 \mathrm{~nm}$ (工艺 3）。不同碱蚀时长处理后, 两 种工艺处理的通道内壁粗糙度相当。因此适当缩短 碱腐蚀时间，对通道内壁粗䊁度影响不大。

\section{3 碱蚀时长对微通道板及制管性能的影响}

首先对两种腐蚀工艺处理后的 MCP 自身电性能 进行对比分析, 如表 3 所示, 相同段号 MCP 采用相 同的氢还原时间，可以看到碱蚀时间较短的工艺 2 , MCP 的平均体电阻略大于腐蚀工艺 3 , 这表明减少 碱腐蚀时长会影响皮料中 $\mathrm{PbO}$ 的还原过程。加载相 同工作电压后，两种腐蚀工艺处理的 $\mathrm{MCP}$ 平均增益 对比可以看出, 腐蚀工艺 2 的 MCP 增益均高于腐蚀 工艺 3 。

对比了两个段号分别采用不同腐蚀工艺下制管 后的测试数据，对不同碱作用时间腐蚀后的微通道 板性能作最终评价。如表 4 所示。可以看到相比于 较长时间的碱蚀工艺（工艺 3 ），缩短碱蚀时长工 艺（工艺 3) 处理的 MCP 在像管测试中展现出更高 的增益性能。而噪声系数相比，两种腐蚀工艺并没 有明显的差异。分析原因，增益的提高主要是由于 缩短碱蚀时长后，通道内部碱金属流失量减少，

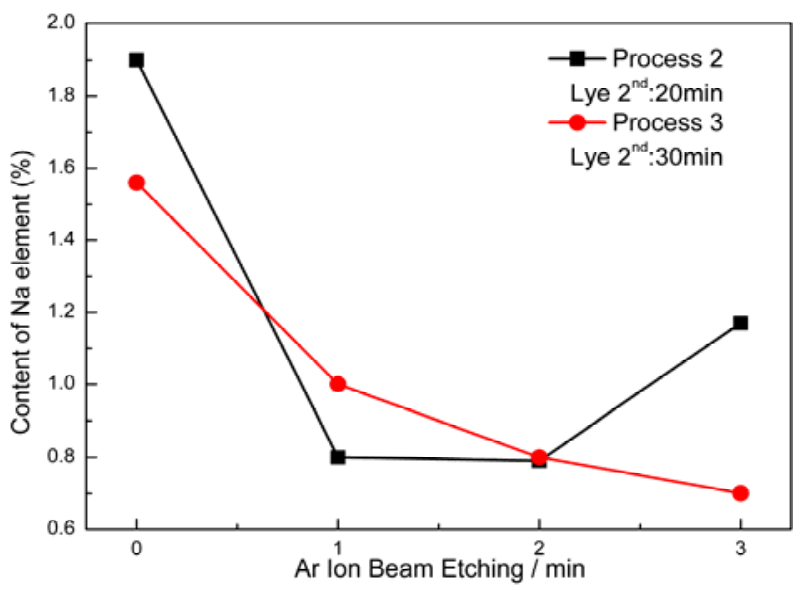

(b) Content distributions of Na element

图 4 两种腐蚀工艺下通道内壁碱金属元素含量随刻蚀时间的变化

Fig.4 The variations of the alkali metals concentration on the MCP cliff with the change of depth with different etching processes

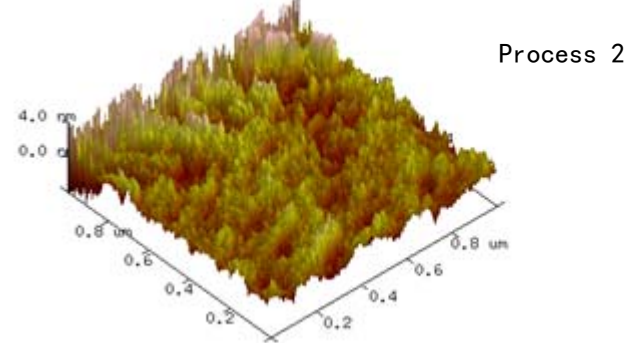

(a) Process 2

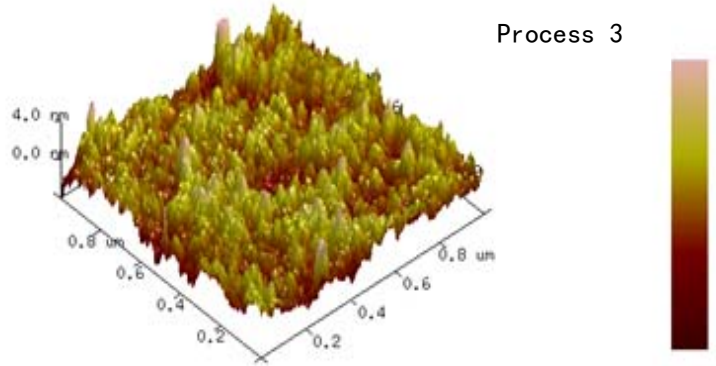

(b) Process 3

图 5 两种腐蚀工艺通道内壁形貌对比

Fig.5 The structure of MCP cliff under different etching processes 
表 2 通道内壁粗糙度对比

Table 2 The coarseness of the channel cliff under different etching processes

\begin{tabular}{ccc}
\hline Parameter & Process 2 & Process 3 \\
\hline Roughness of channel & $0.86 \mathrm{~nm}$ & $0.80 \mathrm{~nm}$ \\
\hline
\end{tabular}

表 3 腐蚀工艺改进后的 MCP 对比测试数据

Table 3 The testing performances of MCP under different etching processes

\begin{tabular}{cccccc}
\hline Number & Etching process & Reduction/min & Gain & Resistance & Quantity \\
\hline \multirow{2}{*}{ DB-1 } & Process 2 & 170 & 15233 & 85 & 27 \\
& Process 3 & 170 & 11265 & 76 & 25 \\
\hline \multirow{2}{*}{ DB-2 } & Process 2 & 150 & 10383 & 97 & 26 \\
& Process 3 & 150 & 10401 & 92 & 23 \\
\hline
\end{tabular}

表 4 不同腐蚀工艺 MCP 制管后对比测试数据

Table 4 The testing performance of image intensity tube with MCP under different etching processes

\begin{tabular}{cccccc}
\hline \multirow{2}{*}{ Number } & $\begin{array}{c}\text { Etching } \\
\text { process }\end{array}$ & $\begin{array}{c}\text { Quantity } \\
\text { gain }\end{array}$ & $\begin{array}{c}\text { Average } \\
\text { gain }\end{array}$ & $\begin{array}{c}\text { Quantity } \\
\text { noise coefficient }\end{array}$ & $\begin{array}{c}\text { Average } \\
\text { noise coefficient }\end{array}$ \\
\hline \multirow{2}{*}{ DB-1 } & Process 2 & 21 & 322 & 21 & 1.18 \\
& Process 3 & 25 & 263 & 23 & 1.20 \\
& Process 2 & 21 & 451 & 21 & 1.16 \\
DB-2 & Process 3 & 23 & 411 & 23 & 1.18 \\
\hline
\end{tabular}

二次电子发射系数提高, 与前文结果一致。MCP 噪声系数与内壁粗䊁度和表面碱金属的状态有较大 的关联, 其中内壁粗䊁度增加可能造成其表面较多 的气体吸附, 这些气体在高电压下发生电离并沿电 场的反方向被加速到通道的输入端, 并有机会碰撞 光电阴极或通道内壁导致产生附加电子, 这些附加 电子在通道电场的作用下再次产生附加的二次电 子, 进而带来离子反馈噪声。同样对于附着在通道 表面原子半径较小的碱金属离子, 也会受工作电压 影响, 在通道内壁迁移脱附, 造成类似的离子反 馈。上文结果中看到, 两种腐蚀工艺处理的 MCP, 在像管测试结果中的噪声系数并未提高, 一方面由于通道内壁粗䊁度并没有明显差异, 另一 方面则反映出, 减少碱蚀时长后, 通道内壁表面相 对丰富的碱金属在工作电压下并未发生明显的迁 移。这表明通过减少碱蚀时长的方式来处理微通道 板，不会增加像管在工作条件下的离子反馈。

\section{4 结论}

根据微通道板碱蚀机理, 对不同碱蚀时间处理 后的微通道板性能进行了研究。XPS 的表征和
AFM 粗糙度的测试结果表明, 减少 MCP 的碱蚀时 长，可以有效降低内壁碱金属离子的流失，而对微 通道板内部粗䊁度影响不大。对微通道板电性能以 及制管后的性能测试结果反应出, 适当减少碱蚀时 间可以提高制管增益，而对噪声系数影响较小。因 此通过调整碱蚀时间即可实现在不增加其他风险因 素的情况下，提高 MCP 及制管性能。

\section{参考文献:}

[1] 潘京生. 微通道板及其主要特征性能 [J]. 应用光学, 2004, 25(5): 25-29.

PAN Jingsheng. Microchannel plates and its main characteristics[J]. Journal of Applied Optics, 2004, 25(5):25-29.

[2] BLASS E H, GOURLEY S. Night vision technology update[J]. Armada Inter, 2001, 25(2): 27-37.

[3] 冯刘, 刘晖, 张连东, 等. 电子清刷对双 MCO 像增强器闪炼噪声 的影响[J]. 红外技术, 2013, 35(5): 295-299.

FENG Liu, LIU Hui, ZHANG Liandong, et al. Influence of electron scrubbing on flicker noise of double-MCP image intensifiers[J]. Infrared Technology, 2013, 35(5): 295-299.

[4] UHLIG F, BRITTING A, EYRICH W, et al. Breakthrough in the lifetime of micro channel plate photomultipliers[J]. Nucl. Instrum 
Methods Phys. Res. A, 2015, 787: 105-109.

[5] 文宛生. 铅玻璃微通道板芯料的腐蚀和扫描电镜检测 [J]. 电子科 学学刊, 1987, 9(5): 20-23.

WEN Wansheng. Etching of the glass of $\mathrm{Pb}$ glass microchannel plate and its quality examination[J]. Journal of Electronics, 1987, 9(5): 20-23.

[6] RAJOPADHYE N R, BHORASKAR S V. Electron emission properties of $\mathrm{Pb}$ and $\mathrm{Bi}$ containing glasses[J]. Journal of Non-crystalline Solid, 1988, 105: 179-184.

[7] 严诚, 王益军, 邓广绪. 微通道板腐蚀工艺研究[J]. 半导体技术, 2007, 32(6): 501-504.

YAN Cheng, WANG Yijun, DENG Guangxu. Study on microchannel plate etching technique[J]. Semiconductor Technology, 2007, 32(6): 501-504

[8] 孙忠文, 黄永刚, 贾金升, 等. 酸蚀对微通道板电性能的影响 [J]. 应用光学, 2008, 29(2): 161-165.

SUN Zhongwen, HUANG Yonggang, JIA Jinsheng, et al. Effect of acid etching on electrical performances of microchannel plate[J]. Journal of Applid Optics, 2008, 29(2): 161-165.
[9] 石攀, 黄永刚, 王久旺, 等. 微通道板加工工程中芯皮玻璃界面的 成分扩散[J]. 硅酸盐学报, 2018, 46(5): 700-706.

SHI Pan, HUANG Yonggang, WANG Jiuwang, et al. Compositional diffusion of core and clad glass interface during thermal process of micro-channel plate[J]. Journal of the Chinese Ceramic Society, 2018 46(5): 700-706.

[10] 王益军, 严诚, 邓光绪, 等. 三代微通道板记忆效应研究[J]. 半导 体技术, 2007, 32(7): 598-601.

WANG Yijun, YAN Cheng, DENG Guangxu, et al. Research on the third generation micro-channel plate memory effect[J]. Semiconductor Technology, 2007, 32(7): 598-601

[11] HILL G E. Secondary electron emission and compositional studies on channel plate glass surface[J]. Adv Electron Phys. 1976, 40A: 153-165.

[12] 潘京生. 三代像增强器用微通道板的改进与发展 [J]. 应用光学, 2006, 27(3): 211-215.

PAN Jingsheng. Development of micro-channel plate used for $3^{\text {rd }}$ generation image intensifier[J]. Journal of Applied Optics, 2006, 27(3): 211-215. 